\title{
On the asymptotic reduction of a bifurcation equation for edge-buckling instabilities
}

\author{
Ciprian D. Coman ${ }^{\dagger}$ \\ University of Nottingham, School of Mathematical Sciences, \\ University Park, Nottingham NG7 2RD, UK
}

September 7, 2017

\begin{abstract}
Weakly clamped uniformly stretched thin elastic plates can experience edge-buckling when subjected to a transverse pressure. This situation is re-visited here for a circular plate, under the assumption of finite rotations and negligible bending stiffness in the pre-buckling range. The eigenproblem describing this instability is formulated in terms of two singularly-perturbed fourth-order differential equations involving the non-dimensional bending stiffness $\varepsilon>0$. By using an extension of the asymptotic reduction technique proposed by Coman and Haughton (Acta Mech 185: 179-200, 2006), these equations are formally reduced to a simple second-order ordinary differential equation in the limit $\varepsilon \rightarrow 0^{+}$. It is further shown that the predictions of this reduced problem are in excellent agreement with the direct numerical simulations of the original bifurcation equations.
\end{abstract}

Keywords: thin films, Föppl-von Kármán plate equations, WKB techniques, asymptotic methods.

\footnotetext{
${ }^{\dagger}$ cdc3p@yahoo.com
} 


\section{Introduction}

The presence of localised deformations in thin elastic bodies such as bars, plates and shells, tends to signal the existence of lower-dimensional mathematical structures that can capture the underlying physics of such phenomena. An archetypal example in this direction is the compressed elastic strut on a nonlinear softening foundation, a configuration that is typically described by a fourth-order ordinary differential equation (e.g., [3]). For this particular scenario it is well known that the slow spatial modulation of the post-critical patterns can be explained by means of a second-order GinzburgLandau type differential equation that follows systematically by a routine application of multiple-scale asymptotic methods (e.g., [20, 22]).

A different type of localised deformation is encountered in relation to stress-concentration phenomena in elastic solids. In thin plates and shells such localised stress concentrations appear near boundaries, or around geometrical discontinuities (e.g., holes, cracks, and inclusions), and take the form of adjacent narrow regions where stresses vary sharply. A reasonable approximation of the deformation undergone by a thin shell can sometimes be obtained by neglecting these boundary-layer effects and considering only the so-called membrane (or momentless) response. Obviously, such simplified approaches become questionable or fail when compressive stresses are involved.

Motivated by the experimental evidence reported in [18], Coman \& Haughton [15] carried out a detailed asymptotic analysis of the edge wrinkling experienced by a thin annular elastic plate uniformly stretched by radial displacements imposed along the two circular boundaries. As the inhomogeneous basic state for this situation is readily available from the closed-form solution of a standard (plane stress) Lamé problem, the edge bifurcation was described by the usual fourth-order buckling equation for initially flat plates subjected to in-plane loading (e.g., $[1,25])$. By neglecting the bending boundarylayers in this equation, the entire wrinkling scenario was then reduced to the solution of a secondorder problem whose predictions compared favourably with the full numerical solutions of the original wrinkling equation. This result turned out to be quite general and was later extended to a number of related edge-wrinkling scenarios involving in-plane loading $[6,7,11]$, including some fairly complicated three-point boundary-value problems [5, 14].

In the present investigation we are motivated to explore the applicability of the aforementioned asymptotic reduction strategy to a rather different edge-wrinkling situation, recently studied by Coman et al. $[9,12]$ within the context of the Föppl-von Kármán (FvK) nonlinear plate theory. In this new case, a weakly clamped uniformly stretched circular elastic plate is subjected to a transverse pressure; due to the gradual development of compressive stresses near the edge of the plate, for a critical value of the loading the plate experiences a regular wrinkling pattern confined to that region. The description of this bifurcation requires including finite rotations in the pre-buckling range, and hence leads to nonlinear equations for the basic state (assumed to possess radial symmetry). Furthermore, the out-of-plane character of the pre-buckling state introduces additional complications; for instance, the displacement buckling equations adopted in [9, 12], and originally derived in [4], consisted of a coupled system involving two second-order and one fourth-order differential equations. In a more traditional form, these equations can also be cast as a system of two coupled fourth-order differential equations (e.g., [10]), and it is this latter formulation that is preferred in what follows.

We start our study in $\S 2$ by reformulating the earlier displacement bifurcation equations, recently discussed by Coman \& Bassom [9], in a more compact form that involves only the transverse deflection of the plate together with a stress function. The resulting differential system is further simplified by seeking solutions with separable variables; this leads to two coupled fourth-order ordinary differential equations whose coefficients depend on the solution of Hencky's nonlinear membrane equation [19]. Having formulated the edge-wrinkling problem, we then proceed in $\S 3$ to embark on the investigation of 
the asymptotic structure for the fundamental system of solutions of the aforementioned equations. By making recourse to the asymptotic simplification strategy proposed by Coman \& Haughton [15], the information that transpires from $\S 3$ is subsequently employed in the next section to yield a second-order simplified boundary-value problem. Comparisons between the predictions of this reduced problem and the direct numerical simulations of the original wrinkling equations confirm the accuracy of the results obtained. The paper concludes with a further discussion of the results reported herein and some remarks on their possible extension.

\section{The bifurcation equations}

We consider a circular thin elastic plate of uniform thickness $h>0$ and radius $a>0$ (i.e., $h / a \ll 1$ ), which is initially stretched uniformly before a transverse uniform load $p>0$ is applied to one of its faces. Along its circumference the plate is flexurally clamped, while the in-plane boundary conditions correspond to prescribed (tensile) tractions; this type of constraint is known as weak clamping, and Figure 1 shows schematically the situation we have in mind. As usual, the deformation of this configuration is referred to a cylindrical system of coordinates $(r, \theta, z)$ with the $z$-axis perpendicular to the median plane of the plate, which also contains the origin of the coordinate axes. The linearly elastic material of the plate is characterised by the Youngs' modulus $E$ and the Poisson's ratio $\nu$.

We shall assume that the deformation of the pressurised plate prior to the onset of the instability is not significantly affected by its bending rigidity and possesses axial symmetry. The implications of the first assumption (i.e., nonlinear membrane basic state) have been explored in detail elsewhere [8], while the latter hypothesis is quite common in this type of analysis (e.g., see [4] and [24]).

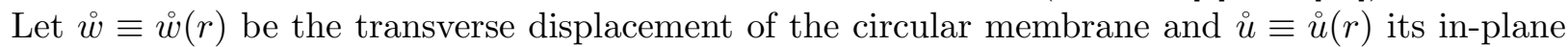
counterpart, where $0 \leq r \leq a$ denotes the radial distance from its centre. Following Campbell [2], if $\stackrel{\circ}{\sigma}_{r r} \equiv \stackrel{\circ}{\sigma}_{r r}(r)$ and $\stackrel{\circ}{\sigma}_{\theta \theta} \equiv \stackrel{\circ}{\sigma}_{\theta \theta}(r)$ represent the incremental stresses produced as a result of applying the transverse pressure $p>0$, then the equations of equilibrium in the radial and normal directions are, respectively,

$$
\stackrel{\circ}{\sigma}_{\theta \theta}=\frac{d}{d r}\left(r \stackrel{\circ}{r r}_{r r}\right) \quad \text { and } \quad\left(\sigma+\stackrel{\circ}{\sigma}_{r r}\right) \frac{d \dot{\circ}}{d r}=-\frac{p r}{2 h} .
$$

The geometrical relations adopted have the well-known form

$$
\stackrel{\circ}{\varepsilon}_{r r}=\frac{d \stackrel{\circ}{d r}}{d r}+\frac{1}{2}\left(\frac{d \stackrel{\circ}{d r}}{d r}\right)^{2}, \quad \stackrel{\circ}{\varepsilon} \theta \theta^{2}=\frac{\stackrel{\leftrightarrow}{r}}{r},
$$

where the strain measures $\stackrel{\circ}{\varepsilon} r r, \stackrel{\circ}{\varepsilon \theta \theta}_{\theta \theta}$ are linked to the stresses $\stackrel{\circ}{\sigma r}_{r r}, \stackrel{\circ}{\sigma \theta}_{\theta \theta}$ by the usual Hooke's Law

$$
\stackrel{\circ}{\sigma}_{r r}-\nu \stackrel{\circ}{\sigma}_{\theta \theta}=E \stackrel{\circ}{\varepsilon}_{r r}, \quad \stackrel{\circ}{\sigma}_{\theta \theta}-\nu \stackrel{\circ}{\sigma}_{r r}=E \stackrel{\circ}{\varepsilon}_{\theta \theta} .
$$

It is helpful to rescale the quantities of interest by introducing

$$
\rho:=\frac{r}{a}, \quad \Sigma_{r r}:=\kappa^{-1}\left(\stackrel{\circ}{\sigma}_{r r}+\sigma\right), \quad \Sigma_{\theta \theta}:=\kappa^{-1}\left(\stackrel{\circ}{\sigma}_{\theta \theta}+\sigma\right),
$$

where

$$
\kappa:=\frac{E}{4}\left(\frac{p a}{E h}\right)^{2 / 3} .
$$

On letting $\alpha:=3\left(1-\nu^{2}\right)$ and writing further

$$
f_{0}:=\alpha \Sigma_{r r}, \quad g_{0}:=2 \alpha^{1 / 2}\left(\frac{\grave{w}}{a}\right)\left(\frac{p a}{E h}\right)^{-1 / 3}, \quad h_{0}:=\alpha \Sigma_{\theta \theta},
$$



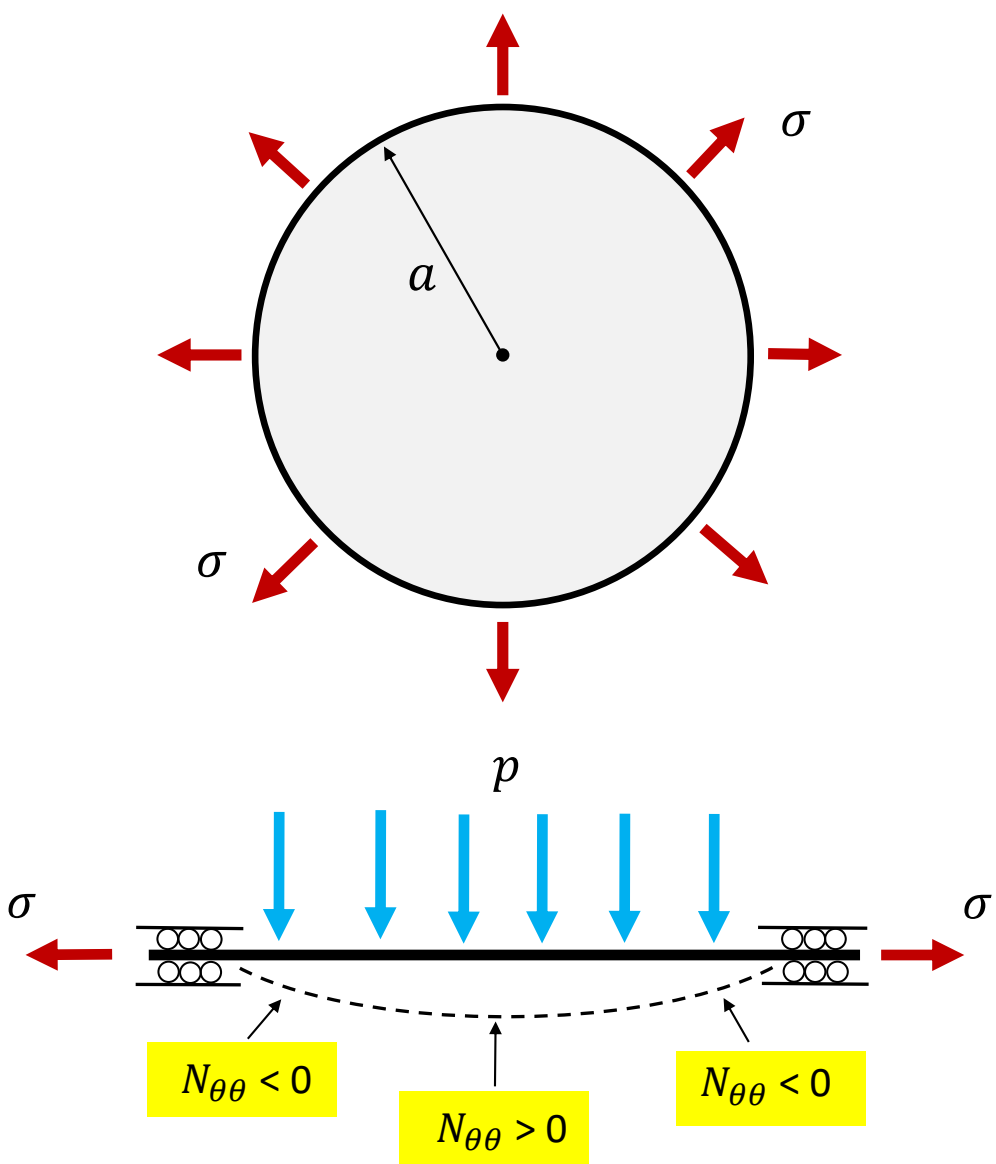

Figure 1: Top and side views of a uniformly stretched circular thin plate subjected to a uniform transverse pressure; the dashed curve shown above represents its deflected shape. For the partial clamping edge conditions adopted in this paper, compressive hoop membrane stresses (i.e., $N_{\theta \theta}<0$ ) develop in a narrow concentric annular region adjacent to the edge of the plate.

elementary algebraic manipulations indicate that these non-dimensional pre-buckling fields satisfy the following equations

$$
\left\{\begin{array}{l}
\frac{d^{2} f_{0}}{d \rho^{2}}+\frac{3}{\rho} \frac{d f_{0}}{d \rho}+\frac{8 \alpha^{3}}{f_{0}^{2}}=0 \\
\frac{d g_{0}}{d \rho}+\frac{4 \alpha^{3 / 2} \rho}{f_{0}}=0 \\
h_{0}=f_{0}+\rho \frac{d f_{0}}{d \rho}
\end{array}\right.
$$

for $0 \leq \rho \leq 1$. Equations (2.7a)-(2.7b) must be solved subject to the requirement that the transverse displacement and the radial stress increment vanish on the outer $\operatorname{rim}(\rho=1)$, and the radial stress is regular near the centre of the plate $(\rho=0)$. These constraints lead to the following boundary conditions

$$
g_{0}(1)=0, \quad f_{0}(1)=4 \alpha \lambda^{-2}, \quad f_{0}^{\prime}(0)=0,
$$


where we have introduced the non-dimensional quantity

$$
\lambda:=\left(\frac{E}{\sigma}\right)^{1 / 2}\left(\frac{p a}{E h}\right)^{1 / 3}
$$

which will serve as the main loading parameter in what follows.

To set up the relevant bifurcation equations, we use the method of adjacent equilibrium in conjunction with the Föppl-von Kármán (FvK) nonlinear plate theory (e.g., see [1, 25]) formulated in terms of the transverse displacement $w$ and a stress function $F$,

$$
D \nabla^{4} w-[F, w]=p \quad \text { and } \quad \nabla^{4} F+\frac{E h}{2}[w, w]=0 .
$$

Here, $D \equiv E h^{3} / 12\left(1-\nu^{2}\right)$ denotes the bending rigidity of the plate, the bracket represents the Monge-Ampère operator defined by $\left[\varphi_{1}, \varphi_{2}\right]:=\left(\nabla^{2} \varphi_{1}\right)\left(\nabla^{2} \varphi_{2}\right)-\left(\boldsymbol{\nabla} \otimes \boldsymbol{\nabla} \varphi_{1}\right):\left(\boldsymbol{\nabla} \otimes \boldsymbol{\nabla} \varphi_{2}\right)$ for any two smooth functions $\varphi_{j}(j=1,2)$, and $F$ is related to the membrane tensor $\boldsymbol{N}$ according to $\boldsymbol{N}=$ $\left(\nabla^{2} F\right) \boldsymbol{I}_{2}-\boldsymbol{\nabla} \otimes \boldsymbol{\nabla} F$, with $\boldsymbol{I}_{2}$ being the usual two-dimensional identity tensor.

We note in passing that the radially symmetric solution discussed above can also be obtained directly from (2.10) by neglecting the bending stiffness of the plate (cf. [24]). As explained in detail in our recent works (e.g, see $[9,10])$, the equations (2.10) must be linearised around the basic state described by the fields $f_{0}, g_{0}, h_{0}$ satisfying (2.7). The outcome can be shown to be a set of two coupled linear partial differential equations for the incremental fields, $\bar{w} \equiv \bar{w}(\rho, \theta)$ and $\bar{F} \equiv \bar{F}(\rho, \theta)$ (say); in the interest of brevity, we suppress the details of these routine calculations.

Motivated by the definition of the pre-buckling state, it helps to rescale the linearised perturbation equations by introducing the non-dimensional quantities

$$
\varepsilon^{2}:=\frac{h}{a}\left(\frac{E}{\sigma}\right)^{1 / 2}, \quad \widehat{w}:=2 \alpha^{1 / 2}\left(\frac{\bar{w}}{h}\right), \quad \widehat{F}:=4 \alpha\left(\frac{\bar{F}}{E h^{3}}\right) .
$$

Since $0<h / a \ll 1$ the above definition of $\varepsilon$ suggests that this quantity is small provided that $E / \sigma \leq \mathcal{O}(1)$. In what follows we shall assume this to be true, and $0<\varepsilon \ll 1$ will be our main asymptotic parameter.

In light of the regular oscillatory deformation pattern experienced by the plate in the azimuthal direction, the aforementioned incremental equations will be solved by seeking solutions with separable variables, that is

$$
\left[\begin{array}{l}
\widehat{w}(\rho, \theta) \\
\widehat{F}(\rho, \theta)
\end{array}\right]=\left[\begin{array}{l}
W(\rho) \\
\Psi(\rho)
\end{array}\right] \sin (m \theta),
$$

for some (as yet) unknown radial amplitudes $W \equiv W(\rho)$ and $\Psi \equiv \Psi(\rho)$. The integer $m>0$ in (2.12), known as the mode number, is determined subject to the requirement that it should render the global minimum of the curve $\lambda$ vs. $m$; we shall rescale this quantity by setting

$$
m^{2}=m_{0} \varepsilon^{-3}, \quad \text { with } \quad m_{0}=\mathcal{O}(1) .
$$

Performing the requisite calculations it turns out that $W$ and $\Psi$ in (2.12) satisfy the linear system

$$
\left[\begin{array}{lc}
\varepsilon \mathcal{L}_{0}^{2}-\lambda^{2} \mathcal{L}_{1} & -\lambda \varepsilon^{2} \mathcal{L}_{2} \\
\lambda \varepsilon \mathcal{L}_{2} & \mathcal{L}_{0}^{2}
\end{array}\right]\left[\begin{array}{l}
W \\
\Psi
\end{array}\right]=\left[\begin{array}{l}
0 \\
0
\end{array}\right]
$$


where we have introduced the differential operators $\mathcal{L}_{j}(j=0,1,2)$,

$$
\begin{gathered}
\mathcal{L}_{0} \equiv \varepsilon^{3} \frac{1}{\rho} \frac{d}{d \rho}\left(\rho \frac{d}{d \rho}\right)-\frac{m_{0}}{\rho^{2}}, \quad \mathcal{L}_{1} \equiv \varepsilon^{3}\left[f_{0}(\rho) \frac{d^{2}}{d \rho^{2}}+\frac{h_{0}(\rho)}{\rho} \frac{d}{d \rho}\right]-\frac{m_{0} h_{0}(\rho)}{\rho^{2}} \\
\mathcal{L}_{2} \equiv \varepsilon^{3} \frac{1}{\rho} \frac{d}{d \rho}\left[\left(\frac{d g_{0}}{d \rho}\right) \frac{d}{d \rho}\right]-\frac{m_{0}}{\rho^{2}}\left(\frac{d^{2} g_{0}}{d \rho^{2}}\right)
\end{gathered}
$$

The system (2.14) must be supplemented with the following boundary conditions (see [9] for a full justification)

$$
W=\Psi=\frac{d W}{d \rho}=\frac{d \Psi}{d \rho}=0, \quad \text { for } \quad \rho=0,1 .
$$

Note that equations (2.14) together with the boundary constraints (2.16) constitute a fairly complicated generalised boundary eigenvalue problem for $\lambda=\lambda\left(m_{0} ; \varepsilon\right)$ where, for a given $\varepsilon, m_{0} \in \mathbb{R}_{+}$ corresponding to the smallest $\lambda>0$ is the one of most significance. Such critical eigenvalues and their corresponding mode numbers will be denoted by $\lambda_{C}$ and $m_{C} \equiv\left(m_{0 C}\right)^{1 / 2} \varepsilon^{-3 / 4}$, respectively; the 'criticality' requirement can be expressed in the compact form

$$
\lambda_{C}:=\min _{m_{0}>0} \lambda\left(m_{0}\right), \quad \text { with } \quad \lambda_{C}=\lambda\left(m_{0 C}\right) .
$$

\section{The asymptotic structure of the solutions for $0<\varepsilon \ll 1$}

Since our main interest is in simplifying the bifurcation equations (2.14) in the limit $\varepsilon \rightarrow 0^{+}$, motivated by the particular structure of those equations, we shall seek solutions of the form

$$
W(\rho)=W_{0}(\rho) \exp \left[S\left(\rho ; \varepsilon^{-1}\right)\right] \quad \text { and } \quad \Psi(\rho)=\Psi_{0}(\rho) \exp \left[S\left(\rho ; \varepsilon^{-1}\right)\right],
$$

with $W_{0}, \Psi_{0}=\mathcal{O}(1)$ and not identically zero, while $S\left(\rho ; \varepsilon^{-1}\right) \gg 1$ for all $\rho \in[0,1]$. All these three functions are yet to be determined, but at this stage our first priority is to understand the dependence of the "phase-function" $S$ on $\varepsilon^{-1} \gg 1$; as it will transpire shortly, the amplitudes $W_{0}$ and $\Psi_{0}$ will be found as a by-product of the same analysis.

On substituting (3.1) into (2.14), at leading order we find that

$$
\left[\begin{array}{ll}
\Delta_{11} & \Delta_{12} \\
\Delta_{21} & \Delta_{22}
\end{array}\right]\left[\begin{array}{l}
W_{0} \\
\Psi_{0}
\end{array}\right]=\left[\begin{array}{l}
0 \\
0
\end{array}\right],
$$

where

$$
\begin{aligned}
& \Delta_{11}:=\varepsilon\left(\varepsilon^{3} p^{2}-\frac{m_{0}}{\rho^{2}}\right)^{2}-\lambda^{2}\left\{\varepsilon^{3} f_{0}(\rho) p^{2}-m_{0}\left[\frac{h_{0}(\rho)}{\rho^{2}}\right]\right\} \\
& \Delta_{12}:=-\lambda \varepsilon^{2}\left\{\varepsilon^{3}\left[\frac{g_{0}^{\prime}(\rho)}{\rho}\right] p^{2}-m_{0}\left[\frac{g_{0}^{\prime \prime}(\rho)}{\rho^{2}}\right]\right\} \\
& \Delta_{21}:=\lambda \varepsilon\left\{\varepsilon^{3}\left[\frac{g_{0}^{\prime}(\rho)}{\rho}\right] p^{2}-m_{0}\left[\frac{g_{0}^{\prime \prime}(\rho)}{\rho^{2}}\right]\right\} \\
& \Delta_{22}=\left(\varepsilon^{3} p^{2}-\frac{m_{0}}{\rho^{2}}\right)^{2}
\end{aligned}
$$


and we have introduced $p \equiv S^{\prime}\left(\rho ; \varepsilon^{-1}\right)$ for notational convenience. The compatibility of the linear homogeneous system (3.2) requires that this latter function satisfies the characteristic equation

$$
\begin{aligned}
H\left(p ; \varepsilon, m_{0}, \rho\right) \equiv \varepsilon\left(\varepsilon^{3} p^{2}-\frac{m_{0}}{\rho^{2}}\right)^{4}-\lambda^{2}\{ & \left.\varepsilon^{3} f_{0}(\rho) p^{2}-m_{0}\left[\frac{h_{0}(\rho)}{\rho^{2}}\right]\right\}\left(\varepsilon^{3} p^{2}-\frac{m_{0}}{\rho^{2}}\right)^{2} \\
& +\lambda^{2} \varepsilon^{3}\left\{\varepsilon^{3}\left[\frac{g_{0}^{\prime}(\rho)}{\rho}\right] p^{2}-m_{0}\left[\frac{g_{0}^{\prime \prime}(\rho)}{\rho^{2}}\right]\right\}^{2}=0 .
\end{aligned}
$$

By using standard arguments (e.g., Newton's polygon [17] or the dominant balance method [21]) we can extract from (3.3) the scaling of $p$ with $0<\varepsilon \ll 1$. It turns out that the characteristic equation has two roots with

$$
\begin{gathered}
p=p_{j} \equiv p_{j 0} \varepsilon^{-2}+p_{j 1} \varepsilon^{-1}+\mathcal{O}(1), \quad(j=1,2), \\
p_{j 0}:=(-1)^{j+1} \lambda f_{0}^{1 / 2}, \quad p_{j 1}:=(-1)^{j+1} \frac{m_{0}\left(2 f_{0}-h_{0}\right)}{2 \lambda \rho^{2} f_{0}^{3 / 2}},
\end{gathered}
$$

and two other roots for which

$$
\begin{gathered}
p=p_{j} \equiv p_{j 0} \varepsilon^{-3 / 2}+p_{j 1} \varepsilon^{-1 / 2}+\mathcal{O}\left(\varepsilon^{1 / 2}\right), \quad(j=3,4), \\
p_{j 0}:=(-1)^{j+1}\left(\frac{m_{0} h_{0}}{\rho^{2} f_{0}}\right)^{1 / 2}, \quad p_{j 1}:=(-1)^{j+1} \frac{m_{0}^{3 / 2}\left(f_{0}-h_{0}\right)^{2}}{2 \lambda^{2} \rho^{3} f_{0}^{5 / 2} h_{0}^{1 / 2}} .
\end{gathered}
$$

In addition to the above, the characteristic equation (3.3) has also two pairs of repeated roots,

$$
p_{5}=p_{6}:=\left(\frac{m_{0}^{1 / 2}}{\rho}\right) \varepsilon^{-3 / 2}+\mathcal{O}(1) \quad \text { and } \quad p_{7}=p_{8}:=-\left(\frac{m_{0}^{1 / 2}}{\rho}\right) \varepsilon^{-3 / 2}+\mathcal{O}(1) .
$$

Letting $W_{j}, \Psi_{j}(j=1,2, \ldots, 8)$ be a fundamental system of solutions of (2.14), next we look for leading-order approximations of these functions corresponding to the roots found above. The solutions associated with (3.4) will be sought with an ansatz of the form

$$
\left[\begin{array}{c}
W_{j}(\rho) \\
\Psi_{j}(\rho)
\end{array}\right]=\left[\begin{array}{c}
W_{0}^{(j)}(\rho) \\
\varepsilon^{2} \Psi_{0}^{(j)}(\rho)
\end{array}\right] \exp \left\{\varepsilon^{-2} \int^{\rho} p_{j 0}(\zeta) \mathrm{d} \zeta+\varepsilon^{-1} \int^{\rho} p_{j 1}(\zeta) \mathrm{d} \zeta\right\}, \quad(j=1,2),
$$

which yields

$$
W_{0}^{(1)}=W_{0}^{(2)} \equiv \rho^{-1 / 2} f_{0}^{-3 / 4} \exp \left\{-\frac{1}{8 \lambda^{3}} \int^{\rho} \frac{m_{0}^{2}}{\zeta^{4} f_{0}^{3 / 2}}\left[1-2 \zeta\left(\frac{f_{0}^{\prime}}{f_{0}}\right)+5 \zeta^{2}\left(\frac{f_{0}^{\prime}}{f_{0}}\right)^{2}\right] \mathrm{d} \zeta\right\}
$$

and

$$
\Psi_{0}^{(j)}=-\frac{g_{0}^{\prime}}{\lambda \rho f_{0}} W_{0}^{(j)} \quad \text { for } \quad j=1,2 .
$$

The characteristic roots (3.5) suggest that the approximations of $W_{3,4}$ and $\Psi_{3,4}$ admit the following representation

$$
\left[\begin{array}{c}
W_{j}(\rho) \\
\Psi_{j}(\rho)
\end{array}\right]=\left[\begin{array}{c}
W_{0}^{(j)}(\rho) \\
\varepsilon \Psi_{0}^{(j)}(\rho)
\end{array}\right] \exp \left\{\varepsilon^{-3 / 2} \int^{\rho} p_{j 0}(\zeta) \mathrm{d} \zeta+\varepsilon^{-1 / 2} \int^{\rho} p_{j 1}(\zeta) \mathrm{d} \zeta\right\}, \quad(j=3,4),
$$


whence, after routine manipulations, we find

$$
W_{0}^{(3)}=W_{0}^{(4)} \equiv \exp \left\{-\frac{1}{4} \int^{\rho} \frac{\zeta}{4 h_{0} f_{0}}\left(f_{0}^{\prime 2}-\frac{8 \alpha^{3}}{f_{0}}\right) \mathrm{d} \zeta\right\}
$$

and

$$
\Psi_{0}^{(j)}=-\frac{\lambda \rho f_{0}\left(h_{0} g_{0}^{\prime}-\rho f_{0} g_{0}^{\prime \prime}\right)}{m_{0}\left(f_{0}-h_{0}\right)^{2}} W_{0}^{(j)} \quad \text { for } \quad j=3,4 .
$$

Finally, the solutions associated with the repeated roots (3.6) can be approximated by

$$
\left[\begin{array}{c}
W_{j}(\rho) \\
\Psi_{j}(\rho)
\end{array}\right]=\left[\begin{array}{r}
W_{0}^{(j)}(\rho) \\
\varepsilon^{-2} \Psi_{0}^{(j)}(\rho)
\end{array}\right] \exp \left\{\varepsilon^{-3 / 2} \int^{\rho} p_{j}(\zeta) \mathrm{d} \zeta\right\}, \quad(j=5,6,7,8) .
$$

This leads to

$$
W_{0}^{(j)}=\frac{\left(\rho g_{0}^{\prime \prime}-g_{0}^{\prime}\right)}{\lambda \rho\left(f_{0}-h_{0}\right)} \Psi_{0}^{(j)} \quad \text { for } \quad j=5,6,7,8
$$

and

$$
\Psi_{0}^{(5)}=\Psi_{0}^{(7)} \equiv \Psi^{I}, \quad \Psi_{0}^{(6)}=\Psi_{0}^{(8)} \equiv \Psi^{I I},
$$

where $\Psi^{j}(j \in\{I, I I\})$ satisfy the second-order order differential equation

$$
\psi^{\prime \prime}-\frac{1}{\rho} \psi^{\prime}-\left[\frac{\left(\rho g_{0}^{\prime \prime}-g_{0}^{\prime}\right)^{2}}{4 \rho^{2}\left(f_{0}-h_{0}\right)}\right] \psi=0 .
$$

All the WKB solutions found above depend on the pre-bifurcation fields $f_{0}, g_{0}$ and $h_{0}$ and their derivatives. Although, strictly speaking, the basic state can only be found by numerically integrating (2.7), historically those equations have been studied extensively using power series. Indeed, if we take

$$
\alpha f_{0}=\sum_{k=0}^{\infty} A_{2 k} \rho^{2 k} \quad \text { and } \quad \alpha^{1 / 2} g_{0}=\sum_{k=0}^{\infty} B_{2 k} \rho^{2 k},
$$

then the coefficients $A_{2 k}, B_{2 k}(k \geq 0)$ can be evaluated recursively as showed in [2, 16, 24]. For our immediate purposes we do not need their precise values, it will suffice to know that none of these quantities is zero. Use of (3.11) in the expression of the WKB solutions indicates that $\lim _{\rho \rightarrow 0^{+}} W_{j}(\rho)<\infty$ for $j=3,4, \ldots, 8$, but the functions $W_{1,2}$ are singular at the origin. Put differently, these latter functions do no represent a good approximation at $\rho=0$. On the other hand, numerical simulations of the bifurcation problem (2.14)-(2.16) suggest that for $\mu \gg 1$ the eigenmodes are strongly localised near the rim of the plate and experience exponential decay within an extended region around $\rho=0$ (cf. $[8,9,12])$. The upshot of this observation is that we can still use the expressions for $W_{1,2}$ found above as long as, instead of enforcing the restraints at $\rho=0$, we apply them at $\rho=\eta$, for some arbitrary $0<\eta \ll 1$.

A number of additional remarks can be made vis-à-vis the WKB approximations derived in this section. We note that the presence of $f_{0} \equiv f_{0}(\rho)$ in the denominator of several of the above expressions raises no concern since $f_{0}(\rho)>0$ for all $\rho \in[0,1]$ (e.g., see [23] and the references therein). The situation is quite different for the (non-dimensional) azimuthal stresses, $h_{0} \equiv h_{0}(\rho)$, which feature in (3.5b). As the loading parameter $\lambda>0$ moves away from zero, azimuthally the plate experiences tension in the central part, while near its edge compression prevails. This amounts to the existence of a unique $\rho_{\#} \in(0,1)$ for which $h_{0}\left(\rho_{\#}\right)=0$, and in the vicinity of this point the expressions (3.8) no 
longer hold. Within the context of the WKB technique this $\rho_{\#}$ is known as a transition point; such a singularity represents an artefact of the integration method rather than of the differential equations themselves. Nevertheless, it is still possible to derive asymptotic expressions away from the transition point, as was done in [15], or one can use the Airy-function uniform asymptotic representations pursued in the related studies $[5,6,11,13]$. Because this aspect is well documented in our previous published work, in the interest of brevity it will not be rehearsed again here; some additional related remarks will appear in the next section.

\section{The asymptotic reduction}

If we let $\boldsymbol{u}:=[W, \Psi]^{T}$, where the superscript ' $T^{\prime}$ stands for matrix transposition, then a fundamental set of solutions of the system (2.14) will consist of the functions $\boldsymbol{u}^{(j)} \equiv\left[W_{j}, \Psi_{j}\right]^{T}(j=1,2, \ldots, 8)$ corresponding to the roots of the characteristic equation (3.3). Thus, we may then represent the general solution of (2.14) in the form

$$
\boldsymbol{u}(\rho)=\sum_{j=1}^{8} C_{j} \boldsymbol{u}^{(j)}(\rho)
$$

where $C_{j} \in \mathbb{R}(j=1,2, \ldots, 8)$ are constants that will be fixed by applying the boundary conditions (2.16). This step yields eight homogeneous equations for the above $C_{j}$-constants, and the existence of a non-trivial solution demands that the coefficient determinant, $\mathcal{D} \equiv \mathcal{D}\left(\lambda ; \varepsilon, m_{0}\right)$ (say), must vanish. Strictly speaking, one has to actually solve the determinantal equation in order to find the eigenvalues $\lambda$ corresponding to (2.14)-(2.16). However, in the limit $\varepsilon \rightarrow 0^{+}$, it is possible to formally simplify this equation by employing the Laplace expansion theorem for determinants (see Appendix A) in conjunction with the asymptotic behaviour of the solutions discussed in $\S 3$. The case of some similar four-by-four determinants associated with edge-wrinkling problems has been discussed in detail by the author in $[6,15]$, while a related eight-by-eight determinantal equation was asymptotically simplified in [14]. In the interest of brevity we omit the routine (but very lengthy) details of the simplification for the determinantal equation studied in this paper, and refer the interested reader to the aforementioned cited works.

Since the value of a determinant is not affected by interchanging rows, we can arrange for the first four rows of our determinant to correspond to the boundary conditions $W(\eta)=W^{\prime}(\eta)=W(1)=$ $W^{\prime}(1)=0$, while the remaining four rows to $\Psi(\eta)=\Psi^{\prime}(\eta)=\Psi(1)=\Psi^{\prime}(1)=0$. It turns out that, to leading order,

$$
\mathcal{D}\left(\lambda ; \varepsilon, m_{0}\right)=\left|\begin{array}{llll}
W_{1}(\eta) & W_{2}(\eta) & W_{3}(\eta) & W_{4}(\eta) \\
W_{1}^{\prime}(\eta) & W_{2}^{\prime}(\eta) & W_{3}^{\prime}(\eta) & W_{4}^{\prime}(\eta) \\
W_{1}(1) & W_{2}(1) & W_{3}(1) & W_{4}(1) \\
W_{1}^{\prime}(1) & W_{2}^{\prime}(1) & W_{3}^{\prime}(1) & W_{4}^{\prime}(1)
\end{array}\right| \cdot\left|\begin{array}{cccc}
\Psi_{5}(\eta) & \Psi_{6}(\eta) & \Psi_{7}(\eta) & \Psi_{8}(\eta) \\
\Psi_{5}^{\prime}(\eta) & \Psi_{6}^{\prime}(\eta) & \Psi_{7}^{\prime}(\eta) & \Psi_{8}^{\prime}(\eta) \\
\Psi_{5}(1) & \Psi_{6}(1) & \Psi_{7}(1) & \Psi_{8}(1) \\
\Psi_{5}^{\prime}(1) & \Psi_{6}^{\prime}(1) & \Psi_{7}^{\prime}(1) & \Psi_{8}^{\prime}(1)
\end{array}\right|+\ldots
$$

where the dots stand for lower-order terms and the expression that has been retained is exponentially dominant. Using exactly the same strategy as in [15] the first determinant can be simplified further and, eventually, the determinantal equation is reduced to

$$
\mathcal{D}\left(\lambda ; \varepsilon, m_{0}\right) \propto\left|\begin{array}{ll}
W_{3}(\eta) & W_{4}(\eta) \\
W_{3}(1) & W_{4}(1)
\end{array}\right|+\ldots=0,
$$


with the dots having the same connotation as already explained. The solutions $W_{3.4} \equiv W_{3,4}(\rho)$ are associated with the roots (3.5); for $j \in\{3,4\}, p_{j 0}$ originate in the differential symbol associated with the operator $\mathcal{L}_{1}$ in $(2.15 \mathrm{a})$, while $p_{j 1}$ involves an additional contribution from the term $(\mathrm{m} / \rho)^{4}$ in $\mathcal{L}_{0}^{2}$. These observations suggest that a leading-order approximation of the eigensystem (2.14)-(2.16) can be provided by the simplified problem*

$$
\left\{\begin{array}{l}
\varepsilon^{3} U^{\prime \prime}+\varepsilon^{3} \mathcal{A}_{1}(\rho) U^{\prime}+\mathcal{A}_{0}\left(\rho ; \lambda, \varepsilon, m_{0}\right) U=0, \\
U(\eta)=U(1)=0
\end{array}\right.
$$

where

$$
\mathcal{A}_{1}(\rho) \equiv \frac{h_{0}(\rho)}{\rho f_{0}(\rho)} \quad \text { and } \quad \mathcal{A}_{0}\left(\rho ; \lambda, \varepsilon, m_{0}\right) \equiv-\frac{m_{0}}{\rho^{2} f_{0}(\rho)}\left[\frac{m_{0} \varepsilon}{\lambda^{2} \rho^{2}}+h_{0}(\rho)\right] .
$$

The equation (4.4a) can be also cast in a more familiar form. Indeed, by recalling the previous scaling (2.13) and letting $R(\rho):=\rho f_{0}(\rho)$, it follows immediately that

$$
U^{\prime \prime}+\left[\frac{R^{\prime}(\rho)}{R(\rho)}\right] U^{\prime}-\frac{m^{2}}{\rho R(\rho)}\left[\frac{\delta^{2}}{\lambda^{2} \rho^{2}}+R^{\prime}(\rho)\right] U=0,
$$

where we have introduced the notations $\mu:=\varepsilon^{-2}$ and $\delta:=m / \mu$. We note in passing that the asymptotic approximations of $W_{3,4}(\rho)$ for $\rho<\rho_{\#}$ and $\rho>\rho_{\#}$, respectively, can be found by using equation (4.5). Its expression is almost identical to equation (6) of [13] (see $\S 3$ in that reference); the only difference lies in the presence of $\lambda^{2}=\mathcal{O}(1)$ which features in the current coefficient of $U$, and the fact that $R(\rho)$ in the present version does not admit an algebraic closed-form expression. Formally, the asymptotic structure of the new equation in the limit $m \gg 1$ is not affected by any of these changes, although the implicit dependence of the basic state (i.e., $R(\rho))$ on $\lambda$ would make the practical implementation of the asymptotic approximation proposed in [13] quite cumbersome. However, it should be clear that solving (4.4) numerically is significantly easier than using the original bifurcation problem (2.14)-(2.16) - obviously, due to the drastic reduction in the order of the corresponding differential equations.

Comparisons between the critical pressures and mode numbers predicted by the reduced equation (4.5) and the direct numerical simulations of the original wrinkling problem are illustrated in Figures 2 and 3; in light of the comments made at the end of the previous section we take the nondimensional parameter $\eta=\mathcal{O}\left(10^{-5}\right)$. Because the effect of the Poisson's ratio is rather weak, we also set $\nu=1 / 3$ in all of our comparisons. In the left window of Figure 2, the round markers identify the minima of the curves $\lambda=\lambda(m)$ obtained from equation (4.5) subject to (4.4b), for $\mu=25 j$ $(j=2,3, \ldots, 8)$; the continuous line represents the corresponding result derived from the numerical integration of the full wrinkling system (2.14)-(2.16). From the relative errors (R.E.) included in the right window it is clear that the agreement between the two sets of results is excellent; for instance, for $\mu=50, R . E . \simeq 1.5 \%$, and this reduces to less than $0.5 \%$ for $\mu=200$. Similar comparisons for the number of wrinkles associated with the onset of the wrinkling instability are recorded in Figure 3; in this case the relative errors between the predictions of the reduced and full models, respectively, vary between $5.7 \%(\mu=50)$ and $3.6 \%(\mu=200)$.

\section{Concluding remarks}

By re-visiting the recent study [8] regarding the edge-wrinkling of uniformly stretched thin elastic plates subjected to a transverse pressure, we have extended the asymptotic reduction strategy proposed by

\footnotetext{
*in the interest of clarity we shall denote the approximation of $W$ by $U$
} 

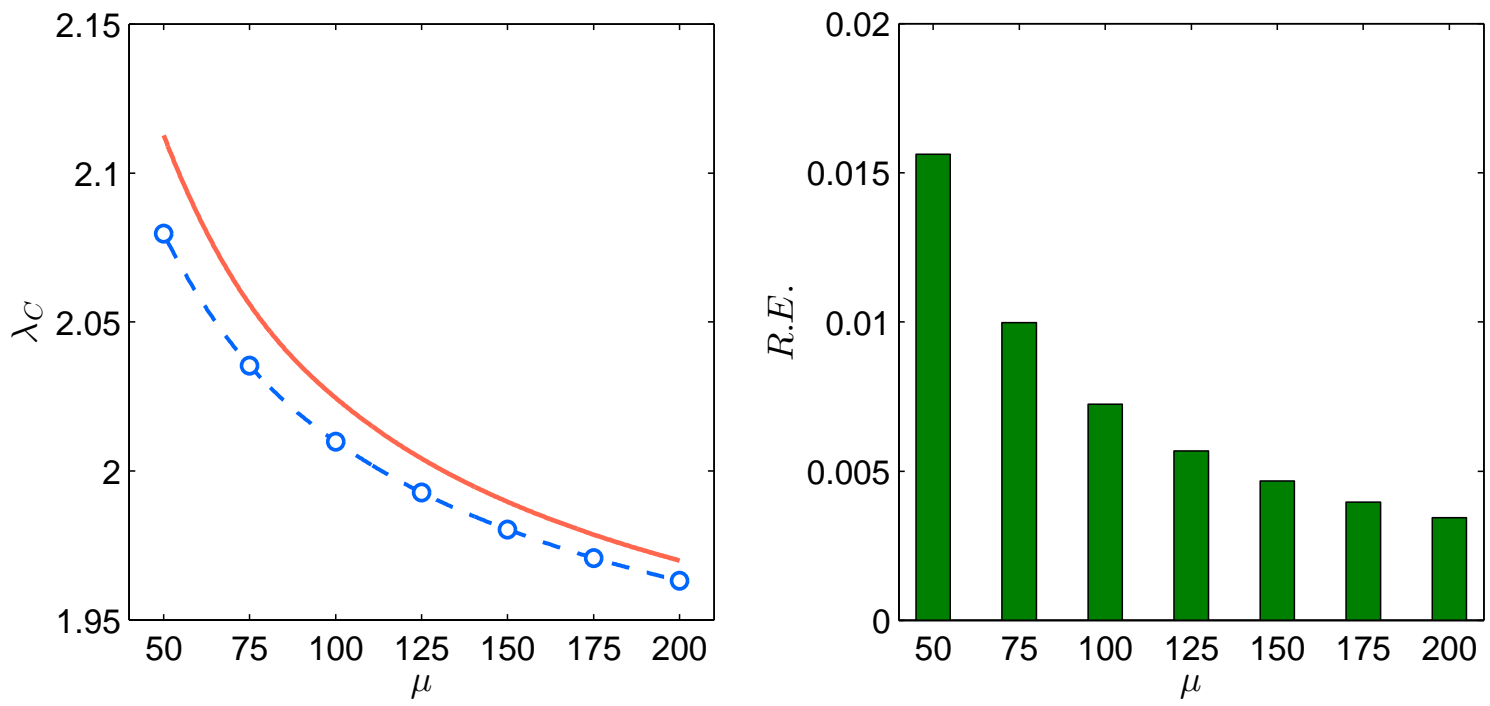

Figure 2: On the left, comparisons between the critical wrinkling load $\lambda_{C}$ given by the reduced problem (4.5) subject to $(4.4 \mathrm{~b})$ and direct numerical simulations of the original boundary-value problem described in $\S 2$ (here $\eta=10^{-5}$ ). The continuous line represents the full wrinkling problem, while the markers and the dashed line indicate the reduced problem. The relative errors $(R E)$ of our comparisons can be seen in the bar plot on the right.
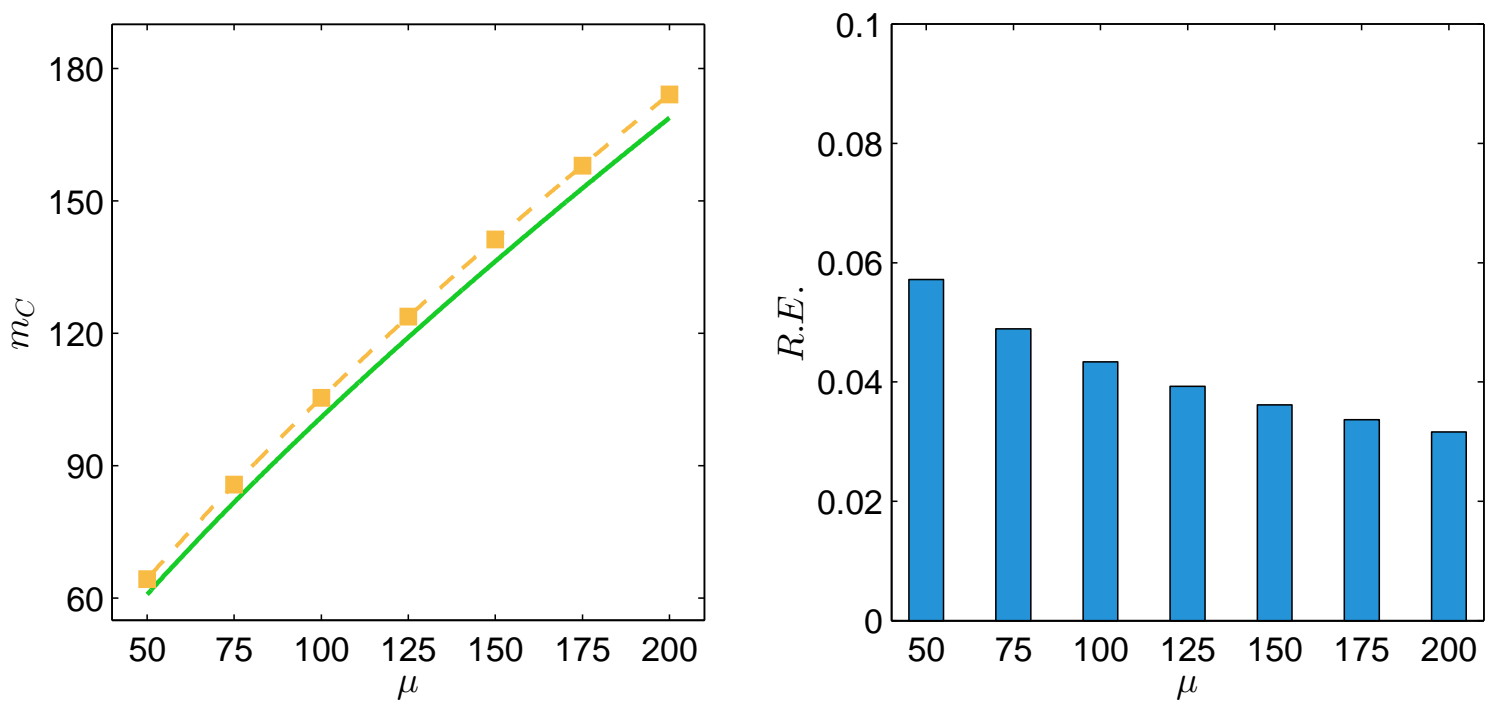

Figure 3: In the left window the critical mode number $m_{C}$ predicted by the reduced problem (4.5) subject to (4.4b) is designated by solid yellow markers, while the continuous line indicates the corresponding values obtained by direct numerical integration of (2.14)(2.16). The relative errors are illustrated on the right.

Coman \& Haughton [15]. Unlike the latter work, in which the basic state was available in closed form and the wrinkling bifurcation was described by a simple fourth-order ordinary differential equation, the present study has demonstrated that those limitations do no represent a serious impediment. 
Indeed, the situation investigated in the previous sections was characterised by a nonlinear basic state which did not admit an analytical representation, while the bifurcation problem consisted of two coupled fourth-order differential equations. The validity of the reduced eigenproblem (4.4) has also been confirmed by comparisons with direct numerical simulations of the original bifurcation system (2.14)-(2.16).

Taken at face value, the conclusion reached by our investigation might seem rather surprising. However, we also draw attention to a number of "clues" that could have anticipated the main findings reported in this work. For instance, the more orthodox boundary-layer approach pursued in [9] and [12], which dealt with similar situations involving a vertical point-load and a uniform transverse pressure, respectively, revealed that the FvK bifurcation system asymptotically decoupled for $\mu \gg 1$, and its behaviour was driven by a hierarchy of second-order ordinary differential equation originating in our operator $\mathcal{L}_{1}-$ see $(2.15 \mathrm{a})$. Furthermore, we note that the wrinkling scenario investigated by Coman \& Haughton in [15] corresponds to letting $\mathcal{L}_{2} \equiv 0$ and setting the multiplicative factor $\lambda$ to unity in (2.14); the effect of these changes leaves the two bifurcation equations trivially decoupled. Of course, this will no longer be true when the plate experiences an out-of-plane deformation prior to the onset of the bifurcation, but the additional solutions introduced by the presence of the second equation in (2.14) will not affect the asymptotic simplification of the original determinantal equation. For this particular reason, the asymptotic limit $\varepsilon \rightarrow 0^{+}$in this study turned out to be the same as in the simpler problem discussed in [15], although the nonlinear basic state still continues to feature in the coefficients of the reduced boundary-value problem (4.4).

In keeping with the related work reported in [8] we have assumed $0<\varepsilon \ll 1$, so that $\lambda=\mathcal{O}(1)$ and $m=\mathcal{O}\left(\varepsilon^{-3 / 2}\right)$. As explained in that reference, for a low or moderate degree of initial stretching it follows that $\varepsilon$ is no longer very small and, in fact, the limits $\varepsilon \gg 1$ or $\varepsilon=\mathcal{O}(1)$ might become relevant (note that in the notation of [12] this corresponds to $0<\mu \ll 1$ and $\mu=\mathcal{O}(1)$, respectively). In that case the roots of the characteristic equation (3.3) are rather different from what we found in $\S 3$, and it can be shown that the asymptotic reduction of $\S 4$ leads to contributions from both equations in (2.14), which can then be combined into a parabolic-cylinder type second-order differential equation. This forms the object of an ongoing investigation whose results will be reported elsewhere in due course.

\section{A Laplace's Expansion Theorem}

For the sake of completeness we include below a short account of the Laplace's Expansion Theorem for determinants.

Let $D$ be the determinant of a square matrix $\boldsymbol{A} \in M_{n \times n}(\mathbb{C})$. If $1 \leq k \leq n$ then a $k$-rowed minor of $D$ is obtained by retaining, with their relative order unchanged, only the elements common to $k$ specified rows and $k$ specified columns. The $k$-rowed minor obtained from $D$ by keeping elements belonging to the rows $r_{1}, r_{2}, \ldots, r_{k}$ and the columns $c_{1}, c_{2}, \ldots, c_{k}$ is denoted by $D\left(r_{1}, \ldots, r_{k} \mid c_{1}, \ldots, c_{k}\right)$.

The cofactor of the minor $D\left(r_{1}, \ldots, r_{k} \mid c_{1}, \ldots, c_{k}\right)$ in the determinant $D$, is defined by

$$
\widetilde{D}\left(r_{1}, \ldots, r_{k} \mid c_{1}, \ldots, c_{k}\right):=(-1)^{r+c} D\left(r_{k+1}, \ldots, r_{n} \mid c_{k+1}, \ldots, c_{n}\right),
$$

where $1 \leq r_{j}, c_{j} \leq n$, with $r_{j} \notin\left\{r_{1}, \ldots, r_{k}\right\}, c_{j} \notin\left\{c_{1}, \ldots, c_{k}\right\}$ for $j=k+1, \ldots, n$, and

$$
r \equiv \sum_{i=1}^{k} r_{i}, \quad c \equiv \sum_{i=1}^{k} c_{i} .
$$


Laplace's Expansion Theorem states that, if $1 \leq r_{1}<\ldots<r_{k} \leq n(1 \leq k<n)$ then

$$
D=\sum_{1 \leq u_{1}<\ldots<u_{k} \leq n} D\left(r_{1}, \ldots, r_{k} \mid u_{1}, \ldots, u_{k}\right) \widetilde{D}\left(r_{1}, \ldots, r_{k} \mid u_{1}, \ldots, u_{k}\right)
$$

\section{References}

[1] D.O. Brush, Bo.O. Almroth, Buckling of Bars, Plates, and Shells, McGraw Hill, New York, 1975 .

[2] J.D. Campbell, On the theory of initially tensioned circular membranes subjected to uniform pressure. Quart. J. Mech. Appl. Math. 9 (1956) 84-93.

[3] A.R. Champneys, G.W. Hunt, J.M.T. Thompson, Localization and Solitary Waves in Mechanics World Scientific, Singapore, 1999.

[4] C.D. Coman, Asymmetric bifurcations in a pressurised circular thin plate under initial tension. Mech. Res. Comm. 47 (2013) 11-17.

[5] C.D. Coman, Some applications of the WKB method to the wrinkling of bi-annular plates in tension. Acta Mech. 224 (2013) 399-423.

[6] C.D. Coman, Edge-buckling in stretched thin films under in-plane bending. Z. angew. Math. Phys. 58 (2007) 510-525.

[7] C.D. Coman, Remarks on elastic buckling for sectorial plates. Int. J. Eng. Sci. 47 (2009) 1002-1013.

[8] C.D. Coman, A.P. Bassom, On the nonlinear membrane approximation and edge-wrinkling. Int. J. Solids Struct. 82 (2015) 85-94.

[9] C.D. Coman, A.P. Bassom, Singularities and wrinkling: the case of a concentrated load. Int. J. Eng. Sci. 106 (2016) 229-244.

[10] C.D. Coman, A.P. Bassom, On the role of in-plane compliance in edge wrinkling. J. Elast. (2016).

[11] C.D. Coman, A.P. Bassom, Wrinkling of pre-stressed annular thin films under azimuthal shearing. Math. Mech. Solids 13 (2008), 513-531.

[12] C.D. Coman, M. Matthews, A.P. Bassom, Asymptotic phenomena in pressurised thin films. Proc. Roy. Soc. Lond. A 471 (2015) 20150471.

[13] C.D. Coman, A.P. Bassom, On the wrinkling of a pre-stressed annular thin film in tension. J. Mech. Phys. Solids (2007) 1601-1617.

[14] C.D. Coman, X. Liu Buckling-resistant thin annular plates in tension. Math. Mech. Solids 19 (2014) 925-951.

[15] C.D. Coman, D.M. Haughton, Localized wrinkling instabilities in radially stretched annular thin films, Acta Mech. 55 (2006) 179-200. 
[16] W.B. Fichter, Some solutions for the large deflections of uniformly loaded circular membranes, NASA Technical Paper 3658.

[17] B.A. Fuchs, V.I. Levin, Functions of a Complex Variable, vol. II. Pergamon Press, 1961.

[18] J.C. Géminard, R. Bernal, F. Melo, Wrinkle formation in axi-symmetrically stretched membranes. Eur. Phys. J. E 15 (2004) 117-126.

[19] H. Hencky, Uber den Spannungszustand in kreisrunden Platten mit verschwindenen Biegungsteifigkeit, Zeitschrift Math. Phys. 63 (1915) 311-317.

[20] C.G. Lange, A.C. Newell The post-buckling problem for thin elastic shells, SIAM J. Appl. Math. 21 (1971), 605-629.

[21] J.A. Murdock, Perturbations: Theory and Methods. John Wiley \& Sons, New York, 1991.

[22] M. Potier-Ferry, Foundations of elastic post-buckling theory. In: J.Arbocz et al. (eds.) Buckling and Post-buckling, Springer Verlag, Berlin (1987).

[23] D.I. Shilkrut, Stability of nonlinear shells. Elsevier Science, Amsterdam, 2002.

[24] J.J. Stoker, Nonlinear Elasticity. Gordon \& Breach, New York, 1968.

[25] H. Troger, A. Steindl, Nonlinear Stability and Bifurcation Theory, Springer-Verlag, Wien, 1991. 